\title{
Elastic Capacitive Tactile Array Pressure Sensor System
}

\author{
Hsing-Cheng Yu, ${ }^{*}$ Chun-Lin Cheng, Pai-Hao Wu, ${ }^{1}$ and $\mathrm{Szu}-\mathrm{Ju} \mathrm{Li}{ }^{1}$ \\ Department of Systems Engineering and Naval Architecture, National Taiwan Ocean University, \\ 2 Pei-Ning Road, Keelung 20224, Taiwan \\ ${ }^{1}$ Material and Chemical Research Laboratories, Industrial Technology Research Institute, \\ 195, Sec. 4, Chung Hsing Road, Chutung, Hsinchu 31040, Taiwan
}

(Received March 1, 2016; accepted March 10, 2017)

Keywords: capacitive array, crosstalk, multipoint measurement, pressure sensor, tactile sensor

Currently, pressure sensors with large areas often use array elements to achieve tactile measurements. Large-area tactile sensors always feature array-type elements connected by wires in series to accomplish extensive ranges of measurements. However, unnecessary noise is generated in the sensors; crosstalk especially is caused by undesired capacitive coupling from array-type element circuits. A grounding method for an elastic capacitive tactile array pressure sensor system (ECTAPSS) is presented in this study to reduce the crosstalk effect of parasitic capacitance. When the capacitive changes influenced by the crosstalk in the floating circuit exceeded $10 \mathrm{fF}$, the crosstalk effect of the ECTAPSS could be reduced by more than $50 \%$. Multipoint measurements of the ECTAPSS were achieved at the same time, and the applied pressures of the ECTAPSS were varied in the range between 0.211 and $0.306 \mathrm{~kg} / \mathrm{cm}^{2}$. Finally, various applied pressure values of the ECTAPSS were measured accurately in multipoint measurements and have been tested and verified under different experimental conditions.

\section{Introduction}

In recent years, wearable electronics have become more and more important in daily life. Physiological data from users may be instantaneously recorded by personal digital assistants. For example, athletes utilize wearable electronic devices to record physiological information and physical activity; moreover, patients with diabetes also use tactile sensor devices to measure foot pressure. Pressure sensors have characteristics similar to human skin. The sensors can learn surface features and other physical information from the contact. For example, they can detect intensity and spatial distribution of force or pressure and other information like shape and position. Large area pressure sensors are usually made of arrays. Measuring the amount of pressure applied to wearable electronics is possible using piezoresistive, piezoelectric, and capacitive sensor technologies. ${ }^{(1)}$ Piezoresistive pressure sensors usually use highly sensitive pressure conductive material to detect pressure changes, for example, a flexible silicon-based tactile sensor. $^{(2)}$ Resistance changes not only with geometry but also with the resistivity of the material. Piezoresistive pressure sensors have a good response mechanically and electrically and have almost no hysteresis. ${ }^{(3)}$ Piezoelectric sensors utilize the positive piezoelectric effect to sense force. ${ }^{(4)}$ For example, the positive force from the foot and shear stress can be acquired by piezoelectric sensors. ${ }^{(5)}$

*Corresponding author: e-mail: hcyu@ntou.edu.tw

http://dx.doi.org/10.18494/SAM.2017.1545 
Capacitive pressure sensors are applications of the principle of parallel-plate capacitance. When pressure is applied to the sensor, the capacitance is changed as a function of changes in the distance between parallel-plate electrodes. Additionally, the change in capacitance is related to the pressure. ${ }^{(6)}$

In applications using wearable electronics as pressure sensors, the design of an elastic capacitive pressure sensor is difficult and outputs are easily influenced by parasitic capacitance. Moreover, the circuitry is also complicated. The sensor has the advantage of high sensitivity, and it is not affected by the external environment. It can be used to continuously measure the static state. In this study, an elastic capacitive tactile array pressure sensor system (ECTAPSS) was designed based on wearable electronics that need to be worn for long periods. The ECTAPSS may be used as a tactile pressure sensor because it is not affected by temperature and is highly accurate.

\section{Capacitance Measurement Circuit}

A trans-impedance amplifier circuit (e.g., current-to-voltage converter) was adopted as a capacitance measurement circuit and is shown in Fig. 1. It is an alternating current (AC) circuit based on a direct measurement method. The equation of input and output can be represented as

$$
\frac{V_{o}}{V_{s}}=-\frac{j \omega R_{f} C_{x}}{j \omega R_{f} C_{f}+1}
$$

where $V_{o}$ is the output signal, $V_{S}$ is the sinusoidal signal of a frequency $f, R_{f}$ is the feedback resistance, $C_{f}$ is the feedback capacitor, $C_{x}$ is the measured capacitance, and $\omega$ is the phase angular frequency of the sinusoidal voltage. The dominant factor in the design is the choice of the feedback resistance $R_{f}$ and the feedback capacitance $C_{f}$. Assuming $\left|j \omega R_{f} C_{f}\right| \gg 1$, Eq. (1) can be simplified as

$$
V_{o}=-\frac{C_{x}}{C_{f}} V_{s}
$$

The amplitude of the AC output signal $V_{o}$ is proportional to the measured capacitance $C_{x}$, and the DC signal can be obtained by a rectifier and a low-pass filter, thereby obtaining the unknown capacitance. The parasitic capacitance $C_{S 1}$ is produced by the direct sine wave voltage source and it cannot affect the capacitance measurement. The parasitic capacitance $C_{S 2}$ is kept at

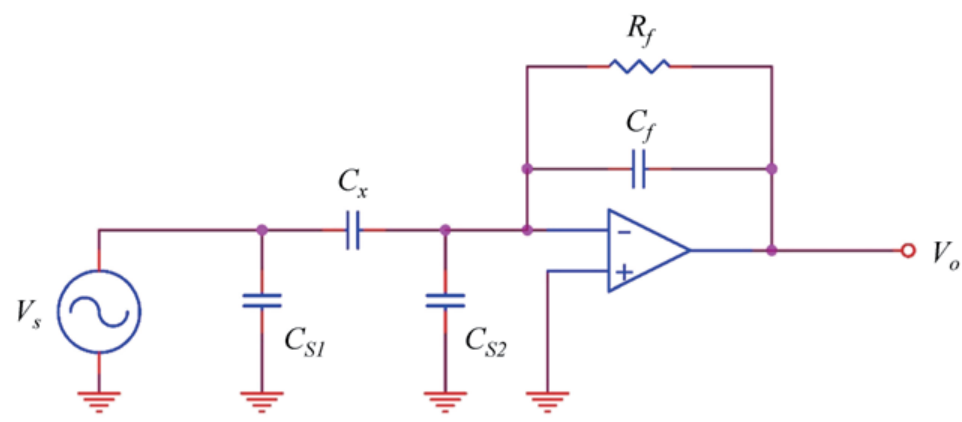

Fig. 1. (Color online) Capacitance measurement circuit. 
virtual ground generated by the operational amplifier; this endpoint voltage is 0 because it does not have a potential difference. Hence, $C_{S 1}$ and $C_{S 2}$ cannot affect the capacitance measurement.

Using a high excitation frequency is the best way to make $\left|j \omega R_{f} C_{f}\right| \gg 1$, which is limited by the bandwidth of the operational amplifier. The main feature of this design is that the sensitivity of the circuit does not depend on the excitation frequency but rather on the excitation voltage and feedback capacitor setting which have high-speed response, parasitic immunity, and low drift characteristics.

\section{System Architecture and Design}

\subsection{Capacitance readout circuit design}

Figure 2 illustrates the capacitance readout circuit. After the sinusoidal signal passes through the measured capacitance and the trans-impedance amplifier, the voltage signal is proportional to the measured capacitance value. Additionally, the output is a DC signal after passing through the rectifier and the filter. Finally, the output signal is acquired by the ADC of the Arduino Due internal function. The measured capacitance can be obtained using Eq. (2).

\subsection{Influence of different feedback capacitance of the circuit}

The sensitivity of the ECTAPSS depends on the voltage of the output signal. The feedback capacitance is an important parameter that affects the converted signals when the input voltage is fixed. Analysis of feedback capacitance for sinusoidal signals is set to $5 \mathrm{~V}_{\mathrm{pp}}$ and $50 \mathrm{kHz}$.

Figure 3 shows the architecture of a parallel-plate ECTAPSS. The upper and lower electrodes are printed circuit boards (PCBs), and the area of the copper electrodes is $0.8 \mathrm{~cm}^{2}$. In the middle is a dielectric material $3 \mathrm{~mm}$ thick with an area $15 \times 15 \mathrm{~mm}^{2}$, a high permittivity of $3.5 \mathrm{~F} / \mathrm{m}$, and very high resilience.

To understand the extent of the capacitance variation based on the sensitivity of the capacitance variation for different feedback capacitors and the variation of pressure as a function of the distance between the layers of dielectric material, the pressure ranges in the experiment environment were varied from 0 to $10 \mathrm{~kg} / \mathrm{cm}^{2}$.

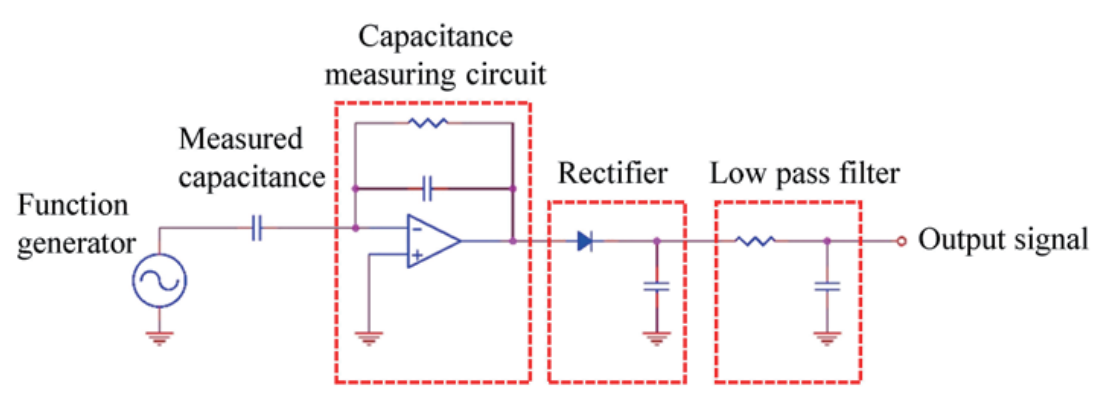

Fig. 2. (Color online) Capacitance readout circuit.

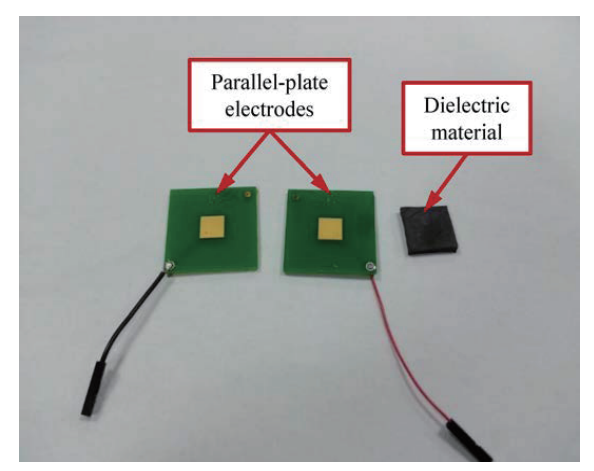

Fig. 3. (Color online) Architecture of parallel-plate capacitive pressure sensor. 
The capacitance variations for various feedback capacitances are large for pressures below 0.5 $\mathrm{kg} / \mathrm{cm}^{2}$, as shown in Fig. 4. The reactive force of the dielectric material is less at values lower than 0.5 $\mathrm{kg} / \mathrm{cm}^{2}$; thus, the variation with distance for the dielectric material is larger. When the feedback capacitance becomes larger, the capacitance variations of parallel-plate ECTAPSS are more obvious. In general, the difference in feedback capacitance between 3 and $4 \mathrm{pF}$ is not obvious, but the magnitude of the difference at $4 \mathrm{pF}$ feedback capacitance is larger than $3 \mathrm{pF}$. Therefore, a $3 \mathrm{pF}$ capacitor was selected as the feedback capacitance in this study.

\subsection{Analysis of filter capacitance}

A filter is a circuit component used to reduce or eliminate interference noise. It determines or eliminates a specific frequency, and the cut-off frequency can be determined by

$$
f_{c}=\frac{1}{2 \pi R_{L} C_{L}}
$$

where $f_{c}$ is the cut-off frequency, $R_{L}$ is the filter resistance, and $C_{L}$ is the filter capacitor.

Because the rectified voltage is a ripple signal rather than stable signal, this study used a lowpass filter to eliminate the ripple signal. When the value of the filter resistance is larger, the presence of filtering interference noise is more obvious. In this study, $1 \mathrm{k} \Omega$ resistance was chosen because a larger resistance made the voltage decrease too obvious.

Different filter capacitors have been designed for experimental analysis. In this experiment, we took $0.5 \mathrm{pF}$ as the measured capacitance and observed output signals of different filter capacitors: 0.001 , 0.01 , and $0.1 \mu \mathrm{F}$. When the filter capacitor was $0.001 \mu \mathrm{F}$, it could not filter a DC signal because its capacitance discharged very quickly and the resultant ripple signals were large. When changed to a $0.01 \mu \mathrm{F}$ filter capacitor, the peak-to-peak signal of the ripple dropped significantly. Although the peak-to-peak ripple improved, the peak-to-peak ripple was still conspicuous. However, the peakto-peak signal of the ripple was significantly improved when a $0.1 \mu \mathrm{F}$ filter capacitor was used.

Figure 5 shows the circuit used for the filter capacitor signal reaction rate test in which the measured capacitors were sequentially switched at 3,2 , and $1 \mathrm{pF}$ per $35 \mu$ s. The filter capacitor $C_{L}$ was 0.01 and $0.1 \mu \mathrm{F}$. Figure 6 shows the results of the filter signal reaction rate test. The response of the filter capacitor at $0.01 \mu \mathrm{F}$ is faster and more stable than the filter capacitor at $0.1 \mu \mathrm{F}$.

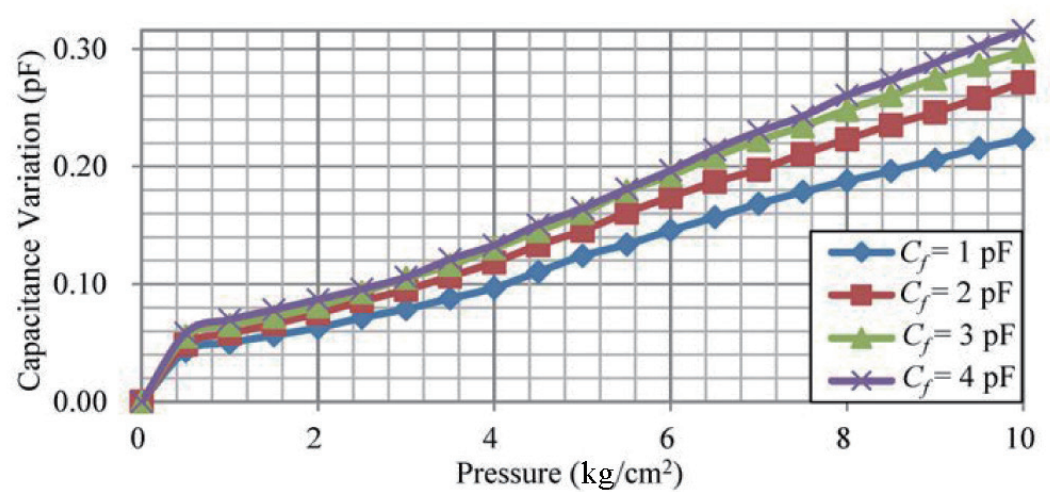

Fig. 4. (Color online) Capacitance variations for various feedback capacitances. 


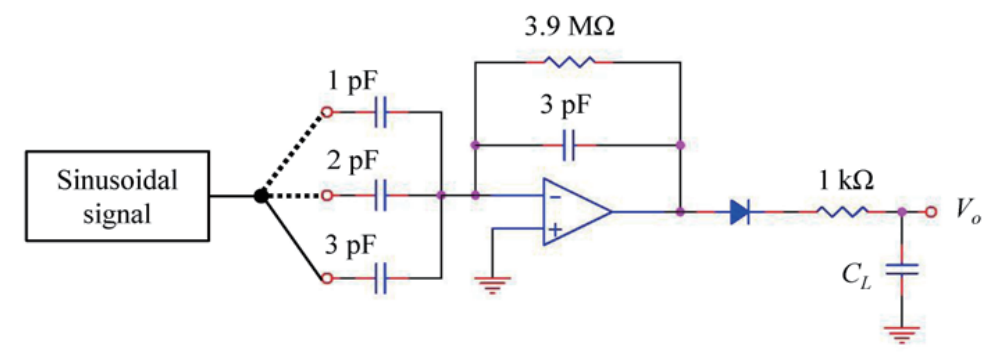

Fig. 5. (Color online) Circuit for the filter capacitor signal reaction rate test.

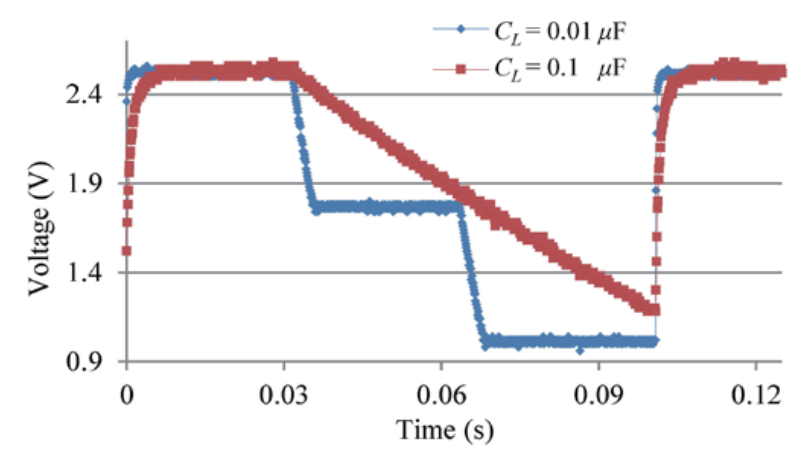

Fig. 6. (Color online) Results of the filter signal reaction rate test.

\subsection{Crosstalk effect and solution of capacitive array}

Parasitic capacitance and crosstalk have been difficult problems in the ECTAPSS. Figure 7(a) shows the capacitive sensing circuit schematic of the ECTAPSS with $3 \times 3$ arrays. The capacitive sensing equivalent circuit became more complex due to crosstalk interference when $\mathrm{C}_{11}$ was the measured capacitance, as shown in Fig. 7(b).

To improve the parasitic capacitance generated by crosstalk, the wires, except for the sinusoidal signal input and output, were connected to ground without presenting a floating state. In Fig. 8(a), capacitances $\mathrm{C}_{22}, \mathrm{C}_{23}, \mathrm{C}_{32}$, and $\mathrm{C}_{33}$ lost their effect after the wires were connected to the ground. The circuit of parasitic capacitance caused by crosstalk was simplified. However, the interference of parasitic capacitance in the simplified circuit increased. The simplified parasitic capacitance circuit and measured capacitance can be considered parallel states as shown in Fig. 8(b).

If the arrays in the ECTAPSS are $n \times n$, the interference in the simplified parasitic capacitance circuit is $2 \times(n-1)$ times the measured capacitance, as shown in Fig. 9(a). In this study, measured capacitance is defined as $C_{M}$, and both sides of the parasitic capacitance passed through simplified circuits defined as $C_{C}$ and $C_{R}$ as shown in Fig. 9(b). Thus, the influence of the parasitic capacitance can be calculated after simplifying the circuit.

Figure 10 shows the capacitive sensor measurement in a single-element circuit. The parasitic capacitance $C_{C}$ has no effect because one of the endpoints is at ground and the other is on the virtual ground of the operational amplifier. The trans-impedance amplifier is a currant-to-voltage converter. The $C_{M}$ and $C_{R}$ cannot interfere with each other because they are at a constant voltage; they only affect the general supply. Therefore, this solution can solve the problem of parasitic capacitance effects. 


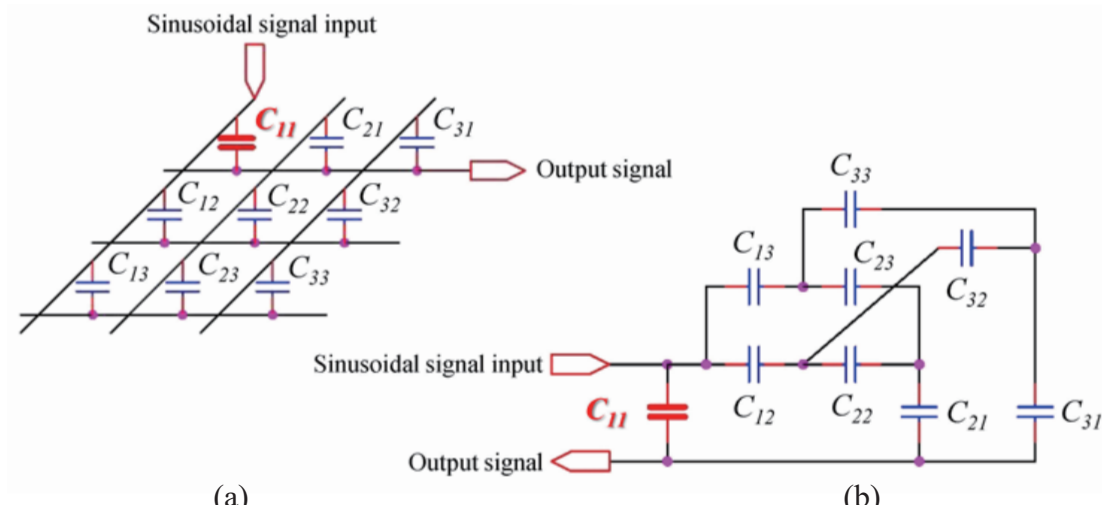

(a)

(b)

Fig. 7. (Color online) The schematic of (a) a capacitive sensing circuit and (b) a capacitive sensing equivalent circuit of the ECTAPSS with $3 \times 3$ arrays.

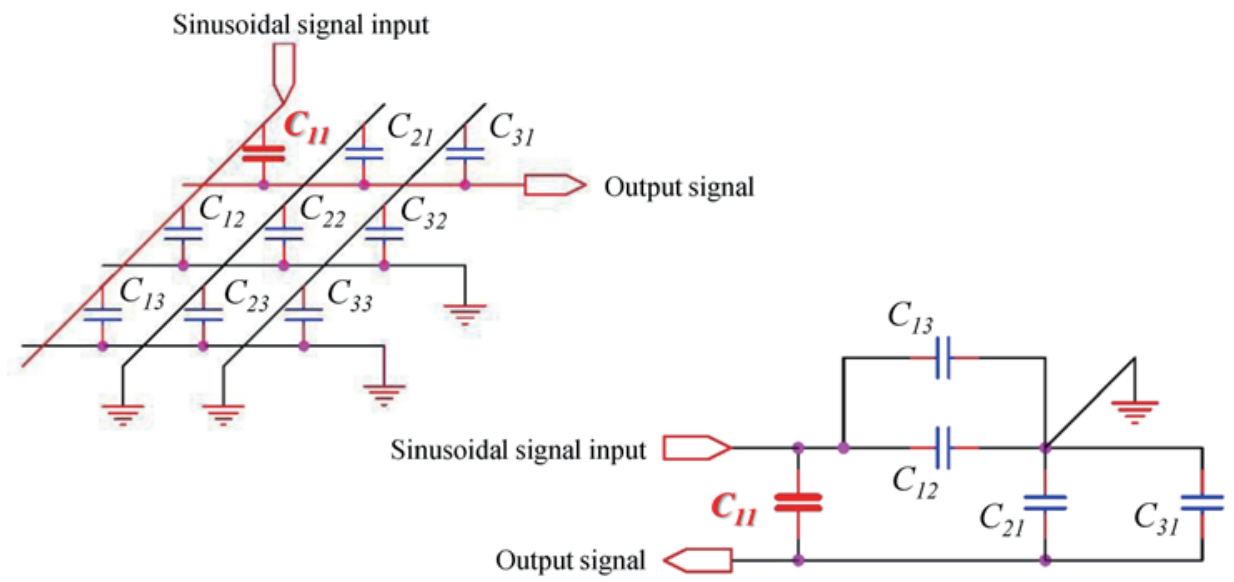

(a)

(b)

Fig. 8. (Color online) The schematic of (a) a capacitive sensing circuit and (b) a capacitive sensing equivalent circuit of the ECTAPSS with $3 \times 3$ arrays after grounding.

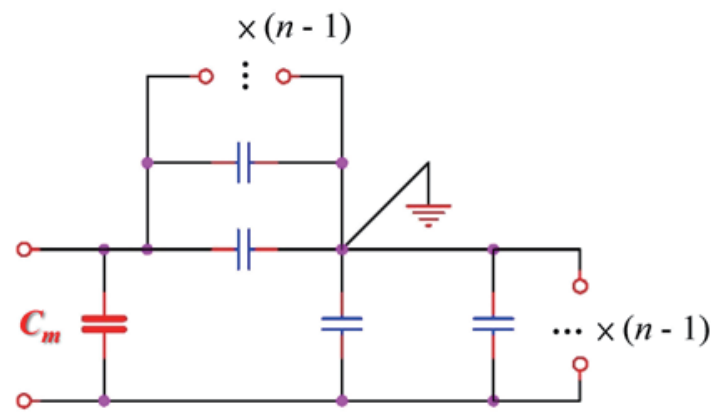

(a)

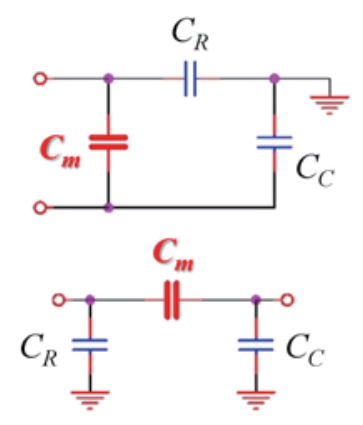

(b)

Fig. 9. (Color online) The schematic of (a) a capacitive sensing circuit and (b) a capacitive sensing equivalent circuit of the ECTAPSS with $n \times n$ arrays after grounding. 


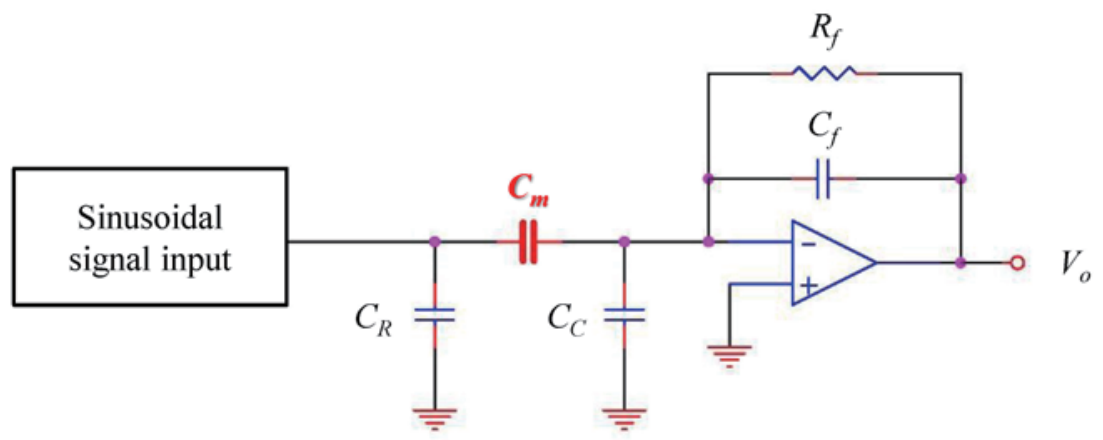

Fig. 10. (Color online) The capacitive sensor measurement in a single-element circuit.

\subsection{ECTAPSS}

Figure 11 is an ECTAPSS with $3 \times 3$ arrays. Because the pressure sensor in this study is based on wearable electronics, the dielectric of the sensor must be elastic so that it can easily acquire the changes associated with different pressures. The lower parallel plate electrode is made of a PCB which contains an array of electrodes. The size of each electrode element is $1 \mathrm{~cm}^{2}$ and the dielectric layer material is located in the middle between the elements.

\subsection{System architecture}

Figure 12 is the block diagram of the designed ECTAPSS. A control multiplexer and desired elements were selected for the Arduino Due so that the sine wave produced by the generator can pass. The converted voltage corresponding to the measured capacitance can be obtained after the trans-impedance amplifier circuit. ${ }^{(7)}$ Finally, the row elements were selected to correspond to the analog/digital converter pin to detect the voltage changes for the measured capacitance. This was calculated using Eq. (2) to obtain the amount of change in the capacitance.

\section{Experimental Results}

\subsection{Circuit comparison}

In this study, the design of the ECTAPSS was based on wearable electronics. To use this system to measure foot pressure, the weight of a person was taken as about $60 \mathrm{~kg}$ and was distributed evenly over both feet. The ECTAPSS had nine capacitive elements, and the area of each capacitive element was $1 \mathrm{~cm}^{2}$. Thus, a capacitive element was exposed to $3 \mathrm{~kg} / \mathrm{cm}^{2}$ pressure, and the maximum pressure rating for each capacitive element in the experimental design was $3 \mathrm{~kg} / \mathrm{cm}^{2}$.

In the floating circuit, the maximum capacitance variations of $\mathrm{C}_{11}$ and $\mathrm{C}_{33}$ with pressure changes were 49 and $68 \mathrm{fF}$, respectively, when the pressure reached $3 \mathrm{~kg} / \mathrm{cm}^{2}$. In the crosstalk suppression circuit, the maximum capacitance variations of $\mathrm{C}_{11}$ and $\mathrm{C}_{33}$ with pressure changes were enhanced to 57 and $72 \mathrm{fF}$, respectively, when the pressure reached $3 \mathrm{~kg} / \mathrm{cm}^{2}$. The capacitance variations generally had to be improved in the crosstalk suppression circuit. The capacitance variations of $C_{11}$ and $C_{33}$ were increased 16.67 and $9.09 \%$, respectively. 


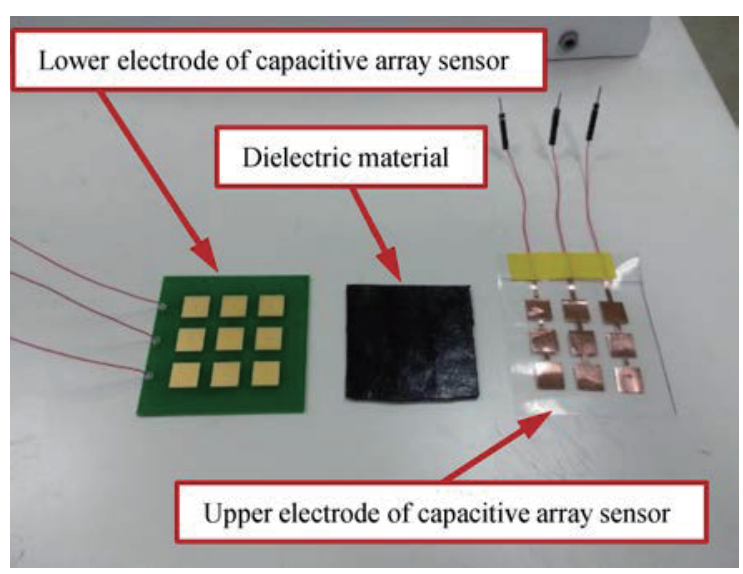

Fig. 11. (Color online) ECTAPSS with $3 \times 3$ arrays.

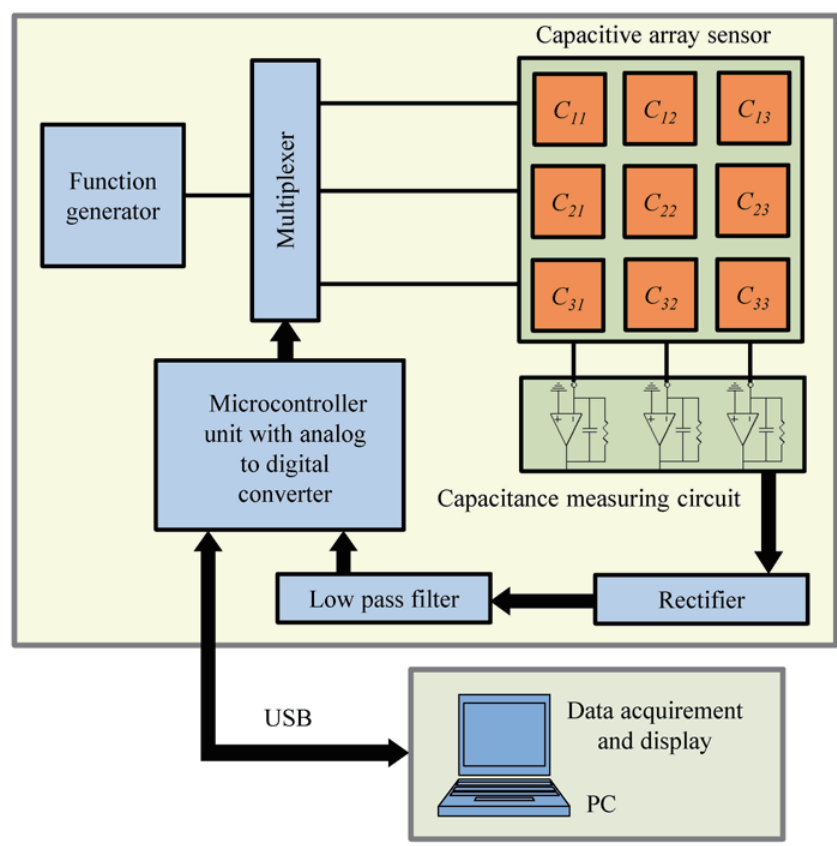

Fig. 12. (Color online) A block diagram of the ECTAPSS.

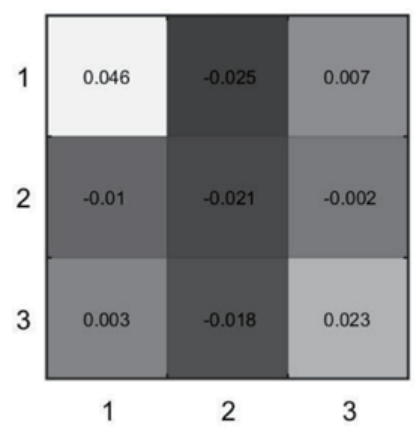

(a)

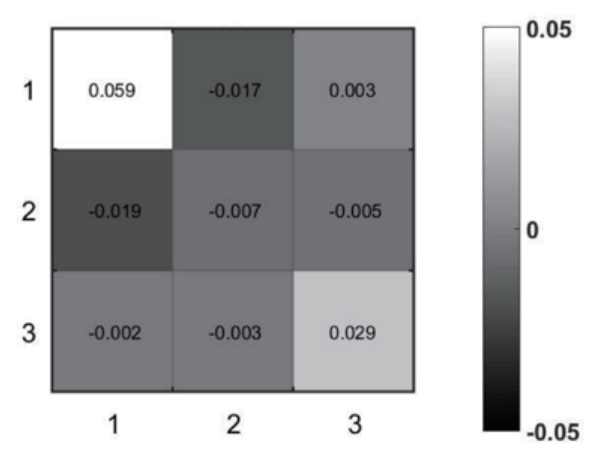

(b)

Fig. 13. Capacitance variations with different two-point pressures in (a) the floating and (b) the crosstalk suppression circuits (unit: $\mathrm{pF}$ ).

Figures 13(a) and 13(b) illustrate the capacitance variations with different two-point pressures in the floating and the crosstalk suppression circuits, respectively. The $\mathrm{C}_{11}$ and $\mathrm{C}_{33}$ have applied pressures of 3 and $1 \mathrm{~kg} / \mathrm{cm}^{2}$, respectively. The variations of capacitance in the floating and the crosstalk suppression circuits can be easily distinguished and correspond to the pressurecapacitance change curve. When the circuit is converted from a floating circuit to a crosstalk suppression circuit, the capacitance variations of elements under pressure rise and the variations in the other elements are significantly reduced. The most obvious change is detected by elements 
$\mathrm{C}_{12}, \mathrm{C}_{22}$, and $\mathrm{C}_{32}$. Although $\mathrm{C}_{33}$ is under pressure causing the material of the dielectric layer to be squeezed and to reduce its capacitance value, its pressure is lower so the variation is smaller. The crosstalk effects in measurement by the floating circuit cause the capacitance variation as measured to be too high. Therefore, the errors caused by crosstalk can be significantly reduced by switching the floating circuit to a crosstalk suppression circuit.

\subsection{Pressure analysis by the ECTAPSS}

To obtain the capacitance changes of each element in the ECTAPSS at different pressures in this study, the elements are subjected to pressures from 0 to $3 \mathrm{~kg} / \mathrm{cm}^{2}$, as shown in Table 1 . In this study, the resolution of the pressure values on each element in the ECTAPSS can be defined as

$$
P_{r}=\frac{P_{v} \times S_{c}}{C_{v}}
$$

where $P_{r}$ is the resolution of the pressure measurement, $P_{v}$ is the pressure value, $S_{c}$ is the sensitivity of the circuit that determines the capacitance oscillation per $5 \mathrm{fF}$, and $C_{v}$ is the capacitance variation. The pressure interval resolution of each capacitive element in the ECTAPSS can be obtained from Eq. (4) and is listed in Table 2. The minimum resolution can be measured to 0.0211 $\mathrm{kg} / \mathrm{cm}^{2}$ when the pressure reaches $3 \mathrm{~kg} / \mathrm{cm}^{2}$. Since the circuit sensitivity set at $5 \mathrm{fF}$, the resolution of the pressure measurement by the capacitance variation is $0.211 \mathrm{~kg} / \mathrm{cm}^{2}$ per $5 \mathrm{fF}$.

In addition to the case of pressure applied to $\mathrm{C}_{11}$ and $\mathrm{C}_{33}$, two different pressure conditions were set on different capacitive elements. In case 1,2 and $1 \mathrm{~kg} / \mathrm{cm}^{2}$ were applied on $\mathrm{C}_{23}$ and $\mathrm{C}_{31}$,

Table 1

Pressure-capacitance variation of each capacitive element (unit: $\mathrm{fF}$ ).

\begin{tabular}{crrrrrrrrr}
\hline Pressure $\left(\mathrm{kg} / \mathrm{cm}^{2}\right)$ & $\mathrm{C}_{11}$ & $\mathrm{C}_{12}$ & $\mathrm{C}_{13}$ & $\mathrm{C}_{21}$ & $\mathrm{C}_{22}$ & $\mathrm{C}_{23}$ & $\mathrm{C}_{31}$ & $\mathrm{C}_{32}$ & $\mathrm{C}_{33}$ \\
\hline 0.0 & 0 & 0 & 0 & 0 & 0 & 0 & 0 & 0 & 0 \\
0.5 & 7 & 9 & 9 & 5 & 6 & 5 & 11 & 8 & 14 \\
1.0 & 16 & 18 & 15 & 15 & 14 & 13 & 23 & 18 & 26 \\
1.5 & 25 & 28 & 23 & 25 & 22 & 22 & 35 & 28 & 37 \\
2.0 & 34 & 38 & 33 & 36 & 31 & 32 & 46 & 36 & 48 \\
2.5 & 45 & 48 & 41 & 47 & 40 & 42 & 58 & 47 & 59 \\
3.0 & 56 & 60 & 51 & 59 & 49 & 51 & 70 & 58 & 71 \\
\hline
\end{tabular}

Table 2

Pressure interval resolution of each capacitive element (unit: $\mathrm{kg} / \mathrm{cm}^{2}$ ).

\begin{tabular}{cccccccccc}
\hline Pressure $\left(\mathrm{kg} / \mathrm{cm}^{2}\right)$ & $\mathrm{C}_{11}$ & $\mathrm{C}_{12}$ & $\mathrm{C}_{13}$ & $\mathrm{C}_{21}$ & $\mathrm{C}_{22}$ & $\mathrm{C}_{23}$ & $\mathrm{C}_{31}$ & $\mathrm{C}_{32}$ & $\mathrm{C}_{33}$ \\
\hline 0.0 & 0.000 & 0.000 & 0.000 & 0.000 & 0.000 & 0.000 & 0.000 & 0.000 & 0.000 \\
0.5 & 0.357 & 0.278 & 0.278 & 0.500 & 0.417 & 0.500 & 0.227 & 0.313 & 0.179 \\
1.0 & 0.313 & 0.278 & 0.333 & 0.333 & 0.357 & 0.385 & 0.217 & 0.278 & 0.192 \\
1.5 & 0.300 & 0.268 & 0.326 & 0.300 & 0.341 & 0.341 & 0.214 & 0.268 & 0.203 \\
2.0 & 0.294 & 0.263 & 0.303 & 0.278 & 0.323 & 0.313 & 0.217 & 0.278 & 0.208 \\
2.5 & 0.278 & 0.260 & 0.305 & 0.266 & 0.313 & 0.298 & 0.216 & 0.266 & 0.212 \\
3.0 & 0.268 & 0.250 & 0.294 & 0.254 & 0.306 & 0.294 & 0.214 & 0.259 & 0.211 \\
\hline
\end{tabular}


respectively. In case 2,1 and $3 \mathrm{~kg} / \mathrm{cm}^{2}$ were applied on $\mathrm{C}_{13}$ and $\mathrm{C}_{32}$, respectively. Figure 14 is the capacitance variation profile under two different pressure conditions.

The pressure value of an element can be obtained in the form of

$$
P_{m}=E\left(\frac{C_{v}}{S_{c}}\right) P_{r}
$$

where $P_{m}$ is the pressure obtained by the ECTAPSS, and $E$ is the integer function of the ratio of the capacitance variation and circuit sensitivity value, which is unconditionally rounded. In this case, a selected pressure range was set at $3 \mathrm{~kg} / \mathrm{cm}^{2}$. The pressure value of an element can be calculated using Eq. (5) with capacitance variation distribution in case 1 and case 2 as shown in Figs. 14(a) and 14(b), respectively. Adopting the pressure interval resolution of each capacitive element in the ECTAPSS from Table 2, the capacitance variations and measured pressure were obtained and are listed in Table 3.

Figure 15 shows the capacitance variation distribution of applied pressure on three capacitive elements. The pressure distribution still responded well after increasing to three capacitive elements, and the others without pressure show less variation. Multipoint measurements as shown in this study are feasible. The results of the three-point pressure measurements are listed in Table 4.

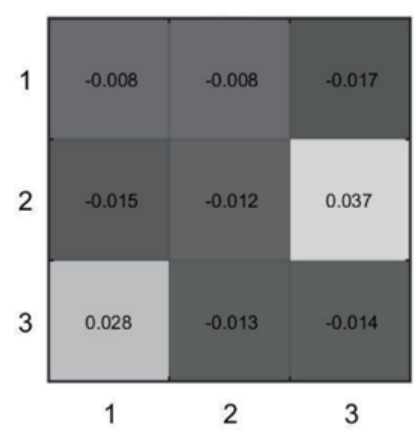

(a)

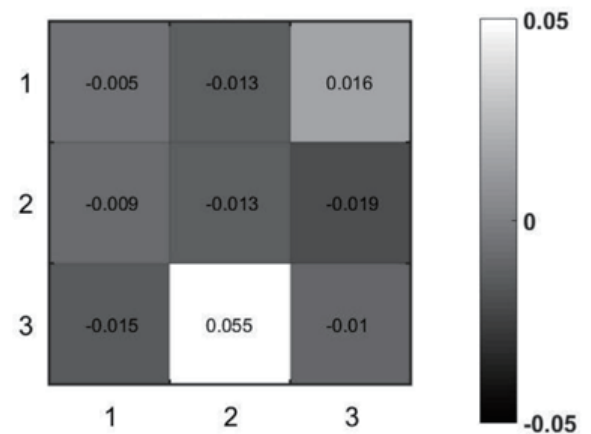

(b)

Fig. 14. Capacitance variation distribution in (a) case 1 and (b) case 2 (unit: pF).

Table 3

Capacitance variation and measured pressure.

\begin{tabular}{lcccc}
\hline Case & $\begin{array}{c}\text { Capacitive } \\
\text { element }\end{array}$ & $\begin{array}{c}\text { Capacitance } \\
\text { variation }(\mathrm{fF})\end{array}$ & $\begin{array}{c}\text { Measured } \\
\text { pressure }\left(\mathrm{kg} / \mathrm{cm}^{2}\right)\end{array}$ & Percent error (\%) \\
\hline \multirow{2}{*}{1} & $\mathrm{C}_{23}$ & 37 & 2.058 & 2.9 \\
\hline \multirow{2}{*}{2} & $\mathrm{C}_{31}$ & 28 & 1.07 & 7.0 \\
\hline & $\mathrm{C}_{13}$ & 16 & 0.882 & 11.8 \\
& $\mathrm{C}_{32}$ & 55 & 2.849 & 5.03 \\
\hline
\end{tabular}




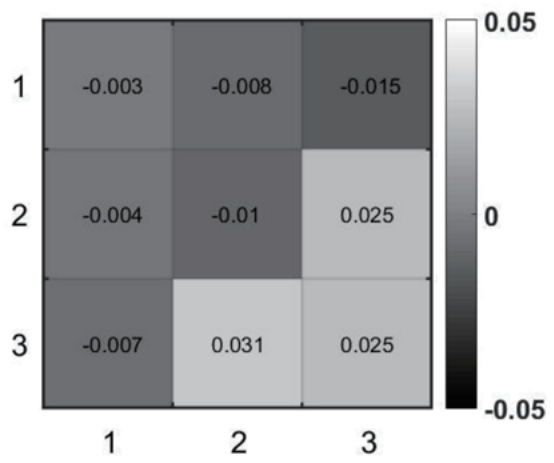

Fig. 15. Capacitance variation distribution of applied pressure on three capacitive elements (unit: pF).

Table 4

Experimental results of the three-point pressure measurements.

\begin{tabular}{ccccc}
\hline $\begin{array}{c}\text { Capacitive } \\
\text { element }\end{array}$ & $\begin{array}{c}\text { Capacitance } \\
\text { variation }(\mathrm{fF})\end{array}$ & $\begin{array}{c}\text { Applied } \\
\text { pressure }\left(\mathrm{kg} / \mathrm{cm}^{2}\right)\end{array}$ & $\begin{array}{c}\text { Measured } \\
\text { pressure }\left(\mathrm{kg} / \mathrm{cm}^{2}\right)\end{array}$ & Percent error (\%) \\
\hline $\mathrm{C}_{23}$ & 25 & 1.5 & 1.47 & 2.0 \\
$\mathrm{C}_{32}$ & 31 & 1.5 & 1.554 & 3.6 \\
$\mathrm{C}_{33}$ & 25 & 1.0 & 1.055 & 5.5 \\
\hline
\end{tabular}

\section{Conclusions}

An ECTAPSS with a minimal parasitic effect is presented in this study as a means to achieve multipoint pressure measurements. The pressure on the capacitive array elements in the ECTAPSS was varied in the range between 0.211 and $0.306 \mathrm{~kg} / \mathrm{cm}^{2}$. Moreover, the error in multipoint measurements at different pressures was less than $11.8 \%$. A simple grounding method presented in this study was applied to the ECTAPSS to solve the problem of parasitic capacitance on crosstalk and to improve the accuracy of the pressure measurements. When capacitance changes were affected by crosstalk in the floating circuits exceeding $10 \mathrm{fF}$, the crosstalk effect in the capacitive array can be reduced by more than $50 \%$.

\section{References}

1 R. E. Saad, A. Bonen, K. C. Smith, and B. Benhabib: The Measurement, Instrumentation and Sensors Handbook, ed. J. G. Webster (CRC Press LLC and IEEE Press, Boca Raton, Florida, 1999) Chap. 25.

2 C. F. Hu, H. Y. Huang, C. C. Wen, L. Y. Lin, and W. Fang: Proc. IEEE Sensors 2010 Conference, eds. T. Kenny and G. Fedder (IEEE, Kona, Hawaii, 2010) p. 1736.

3 L. Wang and D. J. Beebe: Proc. Part of the SPIE Conference on Micromachined Devices and Components IV, eds. P. J. French and K. H. Chau (SPIE, Santa Clara, 1998) p. 359.

4 R. S. Dahiya, M. Valle, G. Metta, L. Lorenzelli, and C. Collini: Proc. 2007 Ph.D. Research in Microelectronics and Electronics Conference (PRIME 2007), eds. D. Belot, T. Taris, and J.-B. Bégueret (IEEE, Bordeaux, 2007) p. 201.

5 S. Kärki, J. Lekkala, H. Kuokkanen, and J. Halttunen: Sens. Actuators, A 154 (2009) 57.

6 C. Liu: Foundations of MEMS, ed. M. Horton (Pearson Education, Inc., New Jersey, 2006) Chap. 4.

7 Y. J. Yang, M. Y. Cheng, W. Y. Chang, L. C. Tsao, S. A. Yang, W. P. Shih, F. Y. Chang, S. H. Chang, and K. C. Fan: Sens. Actuators, A 143 (2008) 143. 